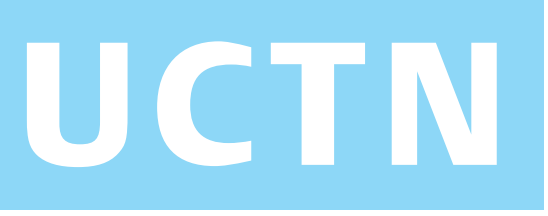

\title{
A rare intestinal malignancy: mantel cell lymphoma
}

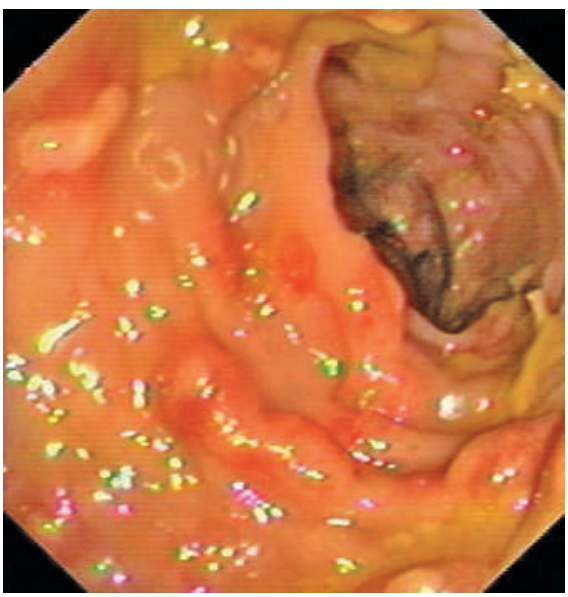

Figure 1 Colonoscopy revealed a large number of different-sized polyps.

Approximately $30 \%$ of primary extranodal lymphomas occur in the gastrointestinal tract. Most of these present as single lesions, although gastrointestinal polyposis occurs in some $10 \%$ of cases, including conditions such as multiple lymphomatous polyposis (MLP) and immunoproliferative small-intestinal disease (IPSID) [1]. MLP is a rare intestinal malignancy characterized by the presence of numerous gastrointestinal polypoid lesions of malignant lymphoma. It is a non-Hodgkin's B-cell lymphoma that is the gastrointestinal counterpart of mantle-cell lymphoma [2]. The polyps range from a few millimeters to several centimeters in size and involve the small and large bowel in $80-90 \%$ of cases and the stomach or duodenum in $50 \%$ of cases [3]. The condition often shows extra-abdominal dissemination, especially to peripheral lymph nodes.

A 68-year-old man presented with a history of abdominal pain, occult lower gastrointestinal bleeding, loss of weight, sideropenic anemia, and fever. Colonoscopy revealed a large number of differentsized polyps (Figure 1). Abdominal ultrasonography showed enlarged retroperitoneal lymph nodes. Chest and abdominal computed tomography (CT) also demonstrated lymphoid dissemination. Gastroscopy and CT enteroclysis did not show

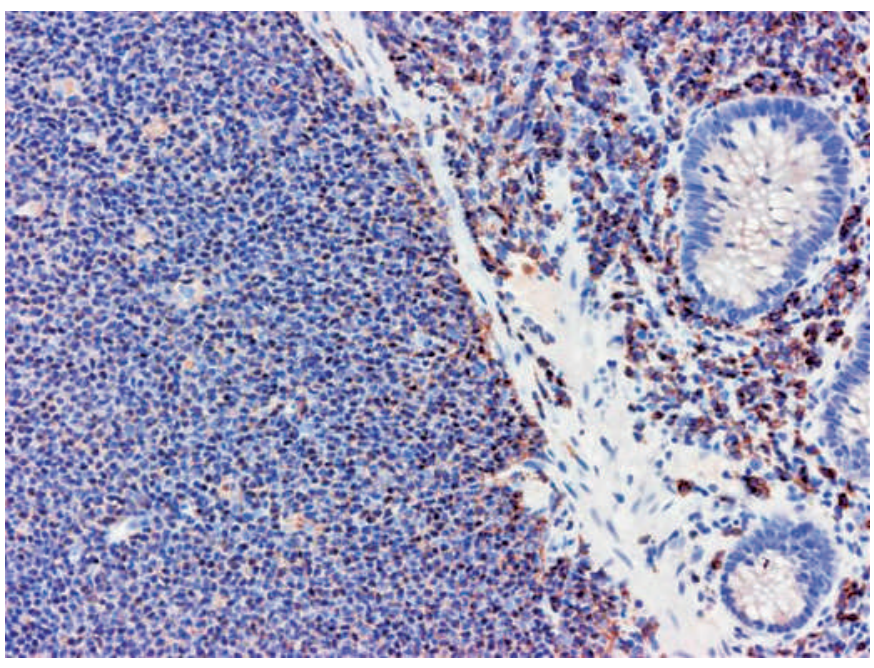

Figure 2 Immunohistochemistry of the tissue samples showed $C D 20$ positivity.

polypoid lesions. Histopathological examination of the large-bowel polyps confirmed lymphocytes with irregular nuclei. Immunohistochemistry (DAKO/ LSAB+DAB) of tissue samples showed CD20, CD5, CD43, and cyclinD1 positivity, and CD79-alpha, CD3, and cytokeratin negativity (Figure 2). Mantle-cell lymphoma was diagnosed on the basis of these data.

The patient underwent four cycles of chemotherapy with a protocol using cyclophosphamide, hydroxydaunomycin, Oncovin (vincristine), and prednisone (CHOP) plus rituximab (Mabthera). The control colonoscopy showed that the polyps had disappeared. At the time of writing, the patient had been asymptomatic and without any abnormal laboratory values for a 12-month follow-up period.

This case represents a rare form of colonic polyposis. Combined chemotherapy was successful and the change in the endoscopic appearance was spectacular. In the management of such rare cases, we recommend careful follow-up of patients by gastroenterologists and oncologists. In case of relapse, repeated chemotherapy or bone-marrow transplantation is needed.

Endoscopy_UCTN_Code_CCL_1AD_2AC
J. Penyige', E. Farczadi², K. Boer², I. Kaszas ${ }^{3}$, J. Csomor ${ }^{4}$, P. Demeter ${ }^{1}$

${ }^{1}$ Dept. of Gastroenterology

2 Dept. of Oncology

${ }^{3}$ Dept. of Pathology, St. Margit Hospital, Budapest, Hungary

${ }^{4}$ Dept. of Pathology, St. Laszlo Hospital, Budapest, Hungary.

\section{References}

${ }^{1}$ Foss HD, Stein H. Pathology of intestinal lymphomas. Recent Results Cancer Res 2000; 156: 33-41

${ }^{2}$ Fernandes BJ, Amato D, Goldfinger M. Diffuse lymphomatous polyposis of the gastrointestinal tract: a case report with immunohistochemical studies. Gastroenterology 1985; 88: 1267-1270

${ }^{3}$ Endoh M, Hiraishi H, Terano A. Gastrointestinal: multiple lymphomatous polyposis. J Gastroenterol Hepatol 1999; 14: 937

Corresponding author

\section{P. Demeter, M.D., Ph.D.}

Dept. of Gastroenterology

St. Margit Hospital

132 Becsi St.

1032 Budapest

Hungary

Fax: $\quad+36-1-4390284$

Email: pal.demeter@sztmargit.hu 